\title{
Extreme Globular Cluster Systems
}

\author{
John P. Blakeslee \\ Department of Physics and Astronomy, Johns Hopkins University \\ Baltimore, $M D$, USA (jpbopha.jhu.edu)
}

\begin{abstract}
The superior resolution and large format of the Advanced Camera for Surveys (ACS) on the Hubble Space Telescope (HST) make it a powerful new tool in the study of extra-galactic globular cluster (GC) systems. We summarize some early results on GC populations from the ACS GTO program, concentrating on the extreme cases of the isolated dwarf NGC 2915 and the core of the massive lensing cluster Abell 1689.
\end{abstract}

\section{Introduction}

ACS has been delivering superb quality images since soon after its installation on HST in March 2002. The earliest ACS science included observations of merging galaxies such as "the Tadpole" (UGC 10214). While Tran et al. (2003) concluded that the bright young star clusters in the Tadpole were unlikely to be young GCs, the ACS science team has a continuing program to study star cluster formation in some star-burst galaxies, including NGC 1569, which is known to harbor massive young clusters that are consistent with being young globulars. Our team has a number of other programs that, while not explicitly designed for studying extra-galactic GCs, have proven to be extremely interesting for this purpose. We discuss two such cases in what follows.

\section{2. "New" Old Globulars in a Dark Matter Dominated Dwarf}

NGC 2915 is an isolated dwarf galaxy about $4.2 \mathrm{Mpc}$ away. While the optical morphology appears to be that of a blue compact dwarf, it is surrounded by a massive Hi spiral disk with an extent more than 5 times greater than the optical light (Meurer et al. 1996). This Hi disk allows for dynamical probing of the dark matter halo to large radii, and the results indicate that NGC 2925 is one of the most dark-matter dominated galaxies known, having $M / L_{B} \sim 80$.

Our 3-band ACS Wide Field Camera images reveal a significant old halo population, including the three globular clusters shown in Figure 1. These objects have colors similar to those of old, metal-poor Galactic globulars. They are 1-2 mag brighter than the mean of the Galactic GC luminosity function, but fainter than the brightest Galactic GCs such as $\omega$ Cen. If we were just extremely lucky with our one ACS pointing and happened to capture all the NGC 2915 GCs in a single frame, then we calculate a specific GC frequency $S_{N} \sim 1$ for this galaxy. However, if we extrapolate according to a more typical power-law GC distribution, then we find a number almost an order of magnitude larger, making NGC 2915 a "high- $S_{N}$ " galaxy. This result is in line with the high gas fraction and $M / L$ ratio, and the apparent scaling of GC population sizes with these quantities, rather than with luminosity, in elliptical galaxies. 

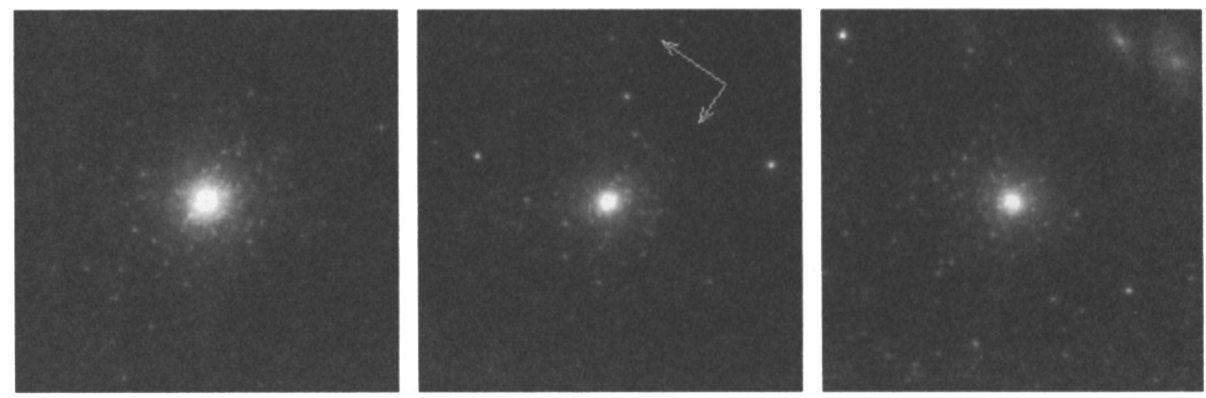

FIG. 1. NGC 2915 globulars newly discovered with ACS.

We can speculate about the future evolution of $S_{N}$ in NGC 2915: whether it will evolve towards typical low values through quiescent star formation in the Hi disk, whether it will remain high or increase through loss of the HI and fading of the stellar light, or how it might change through dissipative merging and starbursts. This may prove a key galaxy for understanding the evolving relationship between GC systems, their host galaxies, and the larger environment.

\section{Abell 1689: The Biggest Globular Population Yet?}

At the other extreme, we have found a teeming GC population in the core Abell $1689 z=0.18$, the most powerful cluster lens in the sky. Rich GC systems were known around many $\mathrm{cD}$ galaxies, but all at $z<0.1$. Besides being the most distant studied to date, extrapolation of the counts indicates this cluster has on the order of $10^{5}$ GCs in its center, or 2-3 times that of any other system and $\sim 7$ times the number found around M87, the prototypical "high- $S_{N}$ " central cluster galaxy. I caution that these results are highly preliminary and we are working to improve the analysis, but it is clearly an extremely rich system.

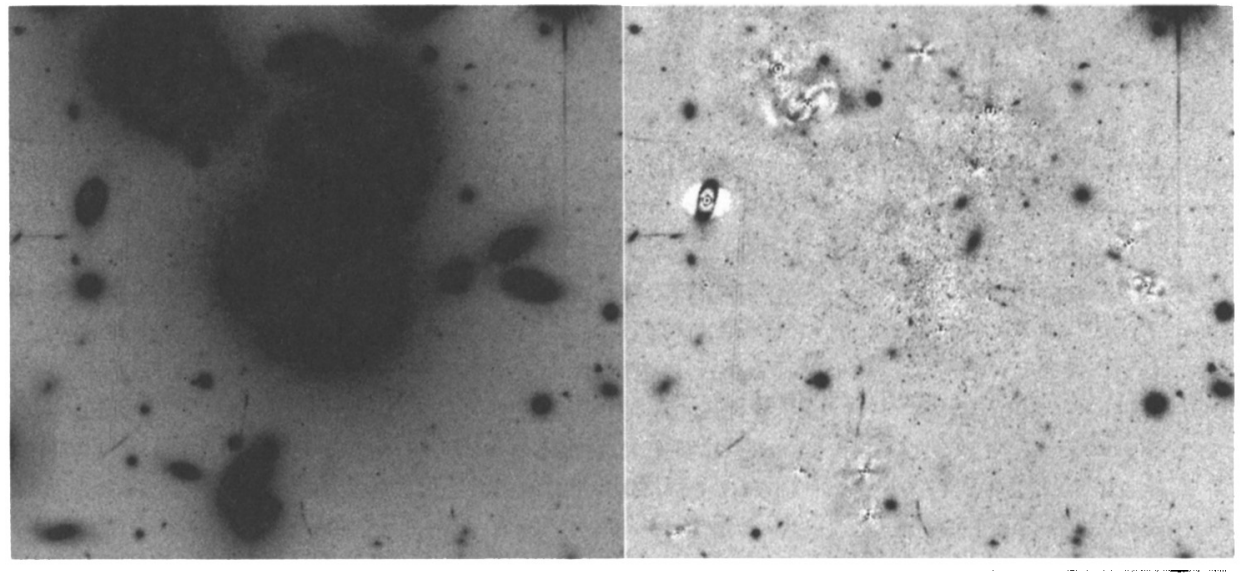

FIG. 2. ACS image of A1689 core (left); faint point sources visible following subtraction (right). These results are all thanks to the devoted efforts of many ACS IDT members.

\section{References}

Meurer, G. R. et al. 1996, ApJ, 111, 1551.

Tran, H. et al. 2003, ApJ, 585, 750. 